\title{
Joint Factor and Kinetic Analysis of Dynamic FDOPA PET Scans of Brain Cancer Patients
}

\author{
N. Dowson ${ }^{1}$, P. Bourgeat ${ }^{1}$, S. Rose ${ }^{3}$, M. Daglish ${ }^{2,3}$, J. Smith ${ }^{2,3}$, M. Fay ${ }^{2}$, \\ A. Coulthard ${ }^{2,3}$, C. Winter ${ }^{2,3}$, D. MacFarlane ${ }^{2}$, P. Thomas ${ }^{2,3}$, \\ S. Crozier ${ }^{3}$, and O. Salvado ${ }^{1}$ \\ 1 The Australian e-Health Research Centre, RBWH, Brisbane, Australia \\ nicholas.dowson@csiro.au \\ 2 Royal Brisbane and Women's Hospital, Herston, Brisbane, QLD, 4029, Australia \\ 3 University of Queensland, RBWH \& St Lucia, Brisbane, Australia
}

\begin{abstract}
Kinetic analysis is an essential tool of Positron Emission Tomography image analysis. However it requires a pure tissue time activity curve (TAC) in order to calculate the system parameters. Pure tissue TACs are particularly difficult to obtain in the brain as the low resolution of PET means almost all voxels are a mixture of tissues. Factor analysis explicitly accounts for mixing but is an underdetermined problem that can give arbitrary results. A joint factor and kinetic analysis is proposed whereby factor analysis explicitly accounts for mixing of tissues. Hence, more meaningful parameters are obtained by the kinetic models, which also ensure a less ambiguous solution to the factor analysis. The method was tested using a cylindrical phantom and the ${ }^{18} \mathrm{~F}$-DOPA data of a brain cancer patient.
\end{abstract}

\section{Introduction}

Glioblastoma multiforme, the most common brain tumour [7], is an aggressive malignancy with most patients dying of the disease in less than a year 4]. Treatment generally involves maximal, safe surgical resection of the tumour followed by chemotherapy and adjuvant radiotherapy [7. Despite this, tumours typically recur within months. The frequency of recurrence could potentially be reduced by specifically targeting regions deemed to contain tumour cells that are likely to proliferate. To delineate the regions containing fecund cells the use of dynamic ${ }^{18}$ F-DOPA Positron Emission Tomography (PET) is being examined as an addition to the Magnetic Resonance (MR) Images, the current gold standard.

The brain is a particularly difficult organ in which to analyse dynamics. Tissues, such as gray matter, are distributed throughout the volume and have a large area of interface with other tissues. This, combined with the low resolution of PET images means that many voxels consist of multiple tissue types. Hence approaches that rely on selecting a volume of interest (VOI) and fitting a predefined kinetic model [3] are challenging. Such an approach has been used for the brain by Schiepers et al., but only to examine the kinetics of the striatum [10]. 
Factor analysis of dynamic images [1] [2] does not assume a particular model and explicitly accounts for mixtures of tissues within voxels. However, even with a positivity constraint, the solution is neither unique nor simple to locate, necessitating the use of $a d-h o c$ explorations of the solution space. Sitek et al. [12] proposed a cost function to search the solution space in a more controlled fashion and make the solution more unique, and hence more physically realistic. Even so, factor analysis has been relegated to delineating VOIs in images from which mean time activity curves (TAC) are extracted to estimate certain functions or model parameters [11] [10]. Also, factor analysis attempts to explain all data while ignoring the effects of noise, so the solution is frequently driven by outliers.

Forcing factors or mean TACs to fit a given model can result in more accurate VOIs, as found by Saad et al. 9. Saad used K-Means clustering rather than factor analysis for region segmentation. Clustering and kinetic analysis were iterated in a two step process. However, K-means clustering assumes voxels have a single label, i.e. they consist of a single tissue, and cluster centres have limited physical meaning, despite fitting a kinetic model, unless priors such as the distribution of TACs and the volume of particular tissue types are known.

This work proposes combining factor analysis and kinetic analysis. Factor analysis explicitly accounts for mixing of tissues, so physically meaningful parameters are obtained by each kinetic model, while kinetic analysis ensures factors give a solution that is unique. This is achieved by minimising the residual between a model of the brain accounting for several tissue types (one compartment model per tissue) and the TAC associated with each factor. In addition, a cost is associated with what percentage of data is explained by the model based on measured image noise. This allows the problem to be naturally formulated within a single cost function, and no weighting factors need to be selected.

The remainder of the paper is organised as follows. The proposed approach is presented in 82 . Some experiments are performed the results of which are discussed in $\$ 3$ before the paper concludes in $\$ 4$.

\section{Methods}

\subsection{Factor Analysis}

Factor analysis is a statistical method used to describe variability among observations using a small number of unseen variables called factors. Here it is assumed that each tissue type can be represented by a single factor, $\mathbf{f}_{p}$, where $\mathbf{f}$ is a vector of intensities at different time points and $p \in\left\{\left[1 ; N_{p}\right] \cap \mathbb{Z}\right\}$ is used to index the factors. The time points, $t_{j}$, are indexed by $j \in\left\{\left[1 ; N_{j}\right] \cap \mathbb{Z}\right\}$, with frame lengths $\Delta t_{j}$. The voxels are indexed by $i \in\left\{\left[1 ; N_{i}\right] \cap \mathbb{Z}\right\}$. The TAC of each voxel is also represented as a vector, $\mathbf{a}_{i}$, representing a single observation of $N_{j}$ variables. The TACs of each voxel are concatenated into an $N_{i} \times N_{j}$ matrix, $A$. Each voxel is formulated as a mixture of factors, i.e. $\mathbf{a}_{i}=\sum_{p=1}^{N_{p}} c_{i p} \mathbf{f}_{p}$ or in matrix form:

$$
A=M F^{T}
$$


where $M$ is a $N_{i} \times N_{p}$ matrix of mixture coefficients and $F$ is a $N_{j} \times N_{p}$ matrix of concatenated factors. Hence factor analysis is the process of finding a valid $M$ and $F$ given $A$, which is an underdetermined problem.

PET data contains substantial amounts of noise, so voxels whose time integral is below some threshold are discarded. Next, the TACs are normalised by their dot product, which is equivalent to removing the proportion of tissue with zero uptake. The normalised TACs are concatenated to form $A$.

A principal component analysis of $A$ is performed, where each principal component is the eigenvector, $\omega_{p}$ of the covariance matrix of $A$. Only the $N_{p}-1$ eigenvectors with the highest eigenvalues, $\lambda_{p}$, are kept. The mean TAC, $\overline{\mathbf{a}}=\frac{1}{N_{i}} \sum_{i} \mathbf{a}_{i}$, is also retained and concatenated with the the eigenvectors to form $W . N_{p}$ may be selected according to how many tissues are expected. The eigenvectors and mean TAC cannot be used as factors as they contain negative elements, which have no physical meaning. Hence, an affine transformation of the eigenvectors is used to obtain positive only factors:

$$
F=\left(\begin{array}{ll}
\mathbf{1} & R^{\prime}
\end{array}\right)\left(\overline{\mathbf{a}} \omega_{1} \ldots \omega_{N_{p}-1}\right)^{T}=R W^{T},
$$

where $R$ is an $N_{p} \times N_{p}$ affine rotation matrix. The values in the $R^{\prime}$ portion of $R$ encode the vertices of a simplex lying on the hyperplane defined by $W$. Similarly $M$ becomes a rotation of principal component coefficients: $M=\left(\mathbf{1} L^{\prime}\right)=L R^{-1}$, where $L^{\prime}$ is (initially) the $N_{i} \times\left(N_{p}-1\right)$ matrix of principal coefficients, so

$$
A=L R^{-1} R W^{T} .
$$

Both $L$ and $R$ are optimised so there are $\left(N_{i}+N_{p}\right)\left(N_{p}-1\right)$ free parameters to obtain from $N_{i} N_{j}$ equations. However $A$ generally only has a rank of around $N_{p}$ or slightly more if the number of factors was underestimated, the system of equations remains under-determined so any solution will not be unique. Enforcing positivity decreases the size of the solution space. Finding the solution using [1] requires an iterative two step approach. $L$ is updated to ensure positivity and $R$ updated. Next $R$ is updated make $F$ 's coefficients positive. This process is dominated by a QR-decomposition each iteration to find $L^{-1} M$, which has complexity $O\left(N_{i} N_{p}^{2}\right)$ [6].

Sitek's approach [12] finds $M$ and $F$ directly, but without care it can converge to the mean TAC with the other factors differing only by small amounts, giving the "low" mixing valued by the cost function in the second step.

\subsection{Kinetic Analysis}

A kinetic model is a linear time invariant model that is fitted to one or more tissues or volumes, which may or may not be independent. Each tissue is assumed to consist of one or more compartments, which are assumed to correspond to physical structures. It is assumed the compartments, $C_{q}$, broadly correspond to vascular, interstitial and inter-cellular spaces. The models for each tissue are by necessity an over-simplification of reality. More complex models would overfit the 
data resulting in unstable and meaningless model parameters. The parameters are symbolised by $k$.

The model parameters are obtained by using least squares techniques to minimise the difference between a simulated TAC and data derived TAC. Standard ordinary differential equation (ODE) solving techniques are used. The computational cost for solving an ODE is $O\left(N_{q} / \epsilon\right)$ [13], where $\epsilon$ is simulation error, $\epsilon$ depends on the amplitude time-step ratio. Hence the complexity is $O\left(N_{q} \frac{\Delta t_{\min }}{f_{\max }}\right)$. An example equation for a 3 compartment model is:

$$
\left(\begin{array}{ccc}
k_{1}-k_{2}-k_{3} & k_{4} \\
0 & k_{3} & -k_{4}
\end{array}\right)\left(C_{1}(t) C_{2}(t) C_{3}(t)\right)^{T}=\frac{\mathrm{d}}{\mathrm{d} t}\left(\begin{array}{l}
C_{2}(t) \\
C_{3}(t)
\end{array}\right)
$$

Alternatively, spectral analysis techniques may also be used, where a TAC is decomposed into a weighted sum of exponential curves. The exponential parameters and weights can be converted into kinetic parameters and the methods have been shown by Gunn et al. to be stable [5], but this technique was deemed too computationally intensive for this work.

For standard least squares techniques an estimate of the (blood) input function, $C_{1}$, is required. This may be estimated directly from measurements of the radioactivity of the patient's blood at different timepoints, but this is invasive. Instead, the less accurate procedure of manually selecting a volume within known cerebral vasculature was taken. Here, the first 7 frames (or 90s) of the dynamic sequence are integrated. This trades off the confounding effects of noise (fewer frames) and metabolism (more frames). The vasculature is then segmented by manually thresholding the image and eroding the result by 2 voxels. The transverse sinus is used as the arteries are narrow and partial volume effects occur.

\subsection{Joint Factor Analysis and Kinetic Analysis}

Factor analysis seeks to obtain a set of pure tissue TACs that lie on a hyperplane in the space of possible TACs. However the locus of TACs obtainable from a given compartment model describes a non-linear $k$-manifold in TAC-space. If neither system noise nor image noise were present and the system was completely described by $N_{p}$ compartment models (one model per factor) then the $N_{p}$ factors would indeed lie on a single $\left(N_{p}-1\right)$-dimensional hyperplane, with samples distributed between factors due to partial voluming effects, resulting in a set of samples enclosed by a simplex. However real data contains substantial amounts of noise and mixing is generally substantial. Hence hyperplanes frequently do not intersect the $\mathrm{k}$-manifold. Unlike standard factor analysis approaches, this work proposes minimising the distance between each factor on the hyperplane and its closest point on the k-manifold:

$$
E_{\text {kinetics }}\left(p, \mathbf{k}_{p}\right)=\frac{1}{N_{j}} \sum_{j}[\underbrace{b_{p}\left(t_{j} ; \mathbf{k}_{p}, C_{1}\left(t_{j}\right)\right)}_{\text {simulated TAC }}-\underbrace{\left(R_{p} W^{T}\right)_{j}}_{\text {hyperplane projection }}]^{2},
$$

where $b_{p}$ is the simulated TAC as a function of $t ; \mathbf{k}_{p}$ and $C_{1}$ are parameters. $R$ is the rotation matrix defined in (3). Each row in $R$ is a single point on the 
hyperplane corresponding to the projection of the simulated TAC $b_{p}$, and defines one factor as described in $\$ 2.1$. The points are easily obtained by whitening the vector of simulated TAC values at timepoints $t_{j}: R \leftarrow\left(\mathbf{1} W^{T} \cdot\left(\mathbf{b}_{p}-\overline{\mathbf{a}}\right)\right)$.

Without further constraints, this approach is dependent on obtaining a good initial choice of parameters and risks the factor converging to a single point, which would describe the data poorly. Hence, an additional constraint is imposed, requiring some fraction of the data to be enclosed by the simplex defined by the $N_{p}$ factors:

$$
E_{\text {data }}=\left[\left[\frac{1}{N_{i}} \sum_{i} \phi\left(\left(\mathbf{a}_{i}-\overline{\mathbf{a}}\right)^{T} \cdot W^{\prime}, R^{\prime}\right)\right]-\alpha_{\text {non-outliers }}\right]^{2},
$$

where $\phi$ acts as a $N_{p}$-dimensional indicator function which returns one for points within the simplex defined by $R^{\prime}$ and zero otherwise. Note the parts of $R$ and $W$ which are not 1 , respectively $R^{\prime}$ and $W^{\prime}$ are used. $\alpha_{\text {non-outliers }}$ is the expected proportion of non-outliers, which is estimated by blurring the probability distribution function (PDF) of samples by a Gaussian kernel defined according to the estimated image noise and measuring the proportion of points that move outside the largest connected populated region of the PDF. All data points within the simplex consist of physically meaningful (positive and less than one) proportions of each factor. Data points outside the simplex have physically non-meaningful mixtures that are assumed to arise from noise. The final objective function is:

$$
\arg \min _{\mathbf{k}} \frac{1}{N_{p}} \sum_{p} E_{\text {kinetics }}+E_{\text {data }}
$$

The algorithm is robust to initialisation, if care is taken to ensure $R^{\prime}$ encompasses most of the data. We select $N_{p}$ points (factors) most distant from the data mean and from each other. Optimal k-parameters are found for each point using the Nelder-Mead algorithm from 10 random initialisations. The best initialisation is iterated to convergence. Both terms in (7) are between 0 and 1 and have similar scales, hence no weighting parameter is used. The largest computational cost arises from solving one set of ODEs per factor every iteration. Around 2 minutes are needed for a system of 3 factors each comprising 3 compartments.

Unlike standard factor analysis, (7) is driven by selecting kinetic parameters, $\mathbf{k}$, not by selecting $R$. Noise is explicitly included in the model and it is assumed some data will not be completely described by the model, and hence avoids being driven by efforts to ensure noisy outliers consist of mixtures that make physical sense. From the point of view of kinetic analysis, prior regions do not need to be selected, and the data best matching a given kinetic model is found. The final solution is also less ambiguous than the solution obtained by simply ensuring positive mixtures and factors.

\section{Experiments and Results}

Proving a particular factor analysis method is superior to another is difficult. Ground truth is unavailable for real data, while simulations cannot currently 


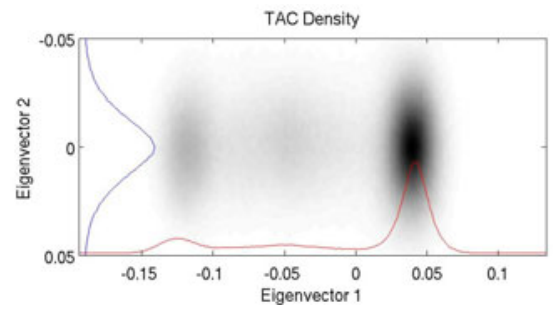

(a) PDF of TACs

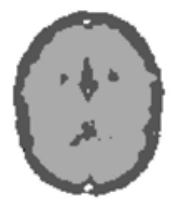

(b) Clustering

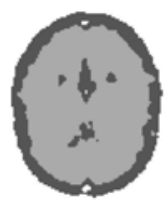

(c) Factor Analysis

Fig. 1. (a) Factor Analysis and (b,c) segmentation of PET SORTEO data

realistically model a true biological system. Even the best available simulations obtained using PET SORTEO, assume only a limited number of factors, differing due to the variation of just one kinetic parameter [8]. This makes testing somewhat artificial as shown in Fig 1, As the TACs in the PET SORTEO data vary across one parameter the locus of the data approximately lies on a line within TAC-space, as shown in Fig. 17. 3 peaks are observable. Standard clustering should perform better than factor analysis in this simple case. However, the central peak has low density compared to its neighbours so similar segmentations are obtained as shown in Fig. 1b and c.

Hence, two data sets are used. The proposed approach is compared to the method proposed by Di Paola [2. Future work will also examine the methods proposed by Sitek and Saad.

- A test sequence using a cylindrical phantom containing a liquid solution into which a radioactive tracer was pumped. Although no ground truth was available, the system was simple: only one free parameter exists, which may be extracted in closed form from the TAC within the phantom.

- A brain scan of a patient suffering from cancer, before surgery. The tumour was distant from the striata (which also takes up FDOPA avidly).

Maximum intensity projections (MIP) of the mixture images from the phantom scans are shown in 2 Images are multiplied by total intensity to prevent noise from obfuscating the result. Both methods identify the input compartment (pipe) and the output compartment (cylinder). Standard factor analysis drives the two factors to the outlying regions of the data. Joint factor and kinetic analysis does not not merely try to encompass all data (and nor does it collapse to a point). Hence it correctly identifies that residual tracer remains in the pipe and has some similarity to the cylinder, giving an above background intensity in the mixture 2 image.

MIPs of the mixtures images for the brain data are shown in Fig. 3, along with the TACs of the 3 factors. Standard factor analysis groups parts of the tumour with the striata, while joint factor and kinetic analysis better separates the two. Both methods separate the tumour into at least two regions, a core and a periphery. Plots on Fig. 3 compare factors to mean TACs of delineations of 


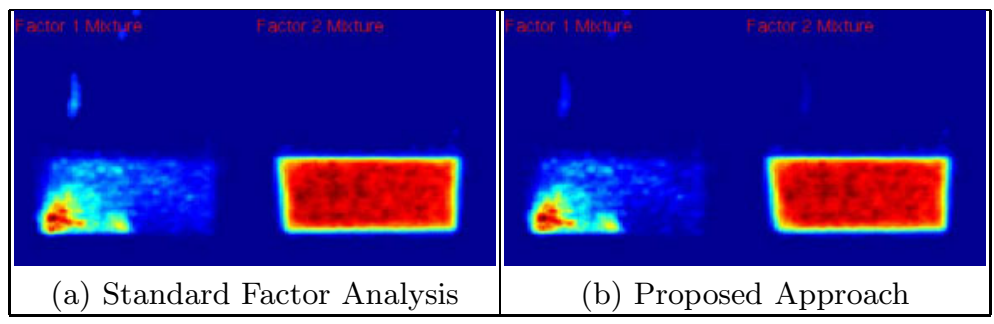

Fig. 2. MIP of mixture images, normalised by intensity, for cylindrical phantom

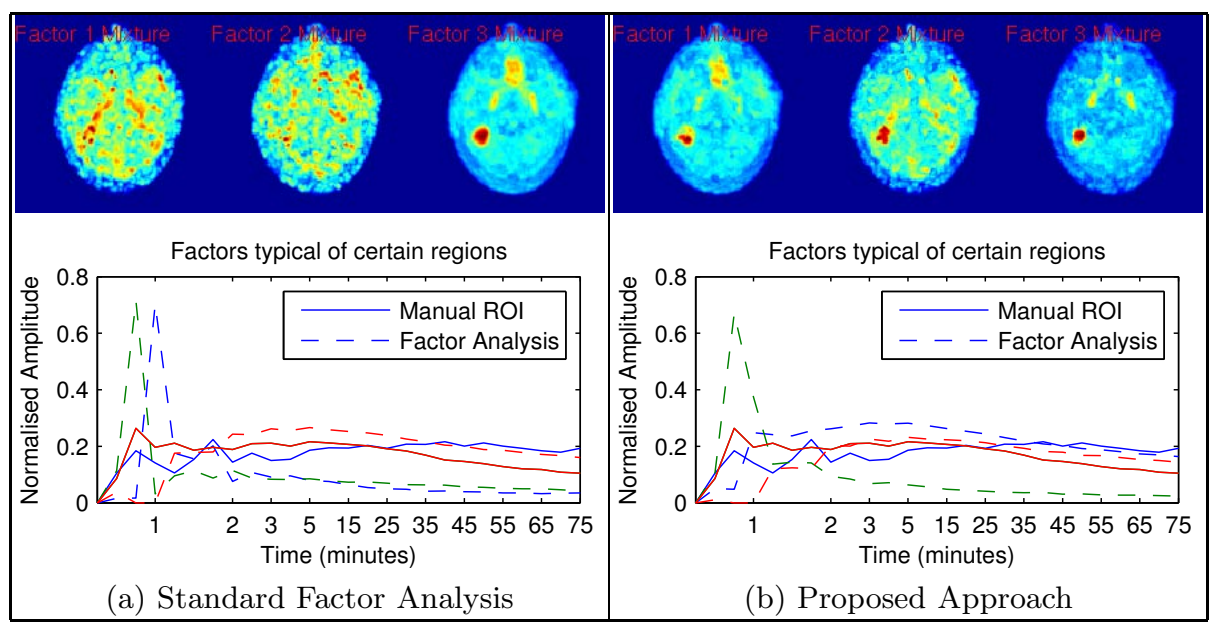

Fig. 3. (Above) MIP of mixture images, normalised by intensity, for pre-surgery test set for 3 factors. (Below) Three model derived TACs for Standard factor analysis and the proposed approach (dashed lines) and two TACs of the manually delineated cerebellum and striata (solid lines). Striata segmented from static PET image at $50 \%$ of maximum. Cerebellum crus obtained by registering PET to 9 labelled atlases and using the majority vote.

the cerebellum crus and the striata. If the joint analysis were better, then two of the factors should better match the two ROIs. In contrast, merely trying to encompass all data gives singular looking curves that do not match ROIs, as shown.

\section{Conclusion}

This work has proposed a method to jointly perform kinetic analysis and factor analysis of dynamic data. This overcomes the chicken and egg problem of existing methods, namely how to select an unknown region in order to accurately obtain 
its characteristic kinetic parameters. A joint approach has the additional advantage of reducing the ambiguity of solutions supplied by existing factor analysis methods. The constraints are not arbitrary, but based on prior knowledge of the system. The proposed approach is efficient because a single cost function is minimised and requires no weight factors to be arbitrarily selected. The proposed method was examined using a real data set and gave hopeful results. Future work will compare the approach to the methods proposed by Sitek and Saad and examine robustness to errors in the estimated arterial input function. The proposed approach will also be extended to constrain the solution further using a Markov Random Field.

\section{References}

1. Barber, D.C.: The use of principal components in the quantiative analysis of gamma camera dynamic studies. Phys. Med. Biol. 25(2), 283-292 (1980)

2. di Paola, R., Bazin, J.P., Aubry, F., Aurengo, A., Cavailloles, F., Herry, J.Y., Kahn, E.: Handling of dynamic sequences in nuclear medcine. IEEE. T. Nuclear Science NS-29(4), 1310-1321 (1982)

3. El Fakhri, G., Sitek, A., Guerin, B., Foley Kijewski, M., Di Carli, M.F., Moore, S.C.: Quantitative dynamic cardiac ${ }^{82} \mathrm{rb}$ pet using generalised factor and compartment analyses. J. Nucelar Medicine 46(8), 1264-1271 (2005)

4. Greene, F.L. (ed.): AJCC Cancer Staging Manual, 6th edn., ch. 47, pp. 387-390. Springer, Heidelberg (2002)

5. Gunn, R.N., Gunn, S.R., Turkheimer, F.E., Aston, J.A.D., Cunningham, V.J.: Positron emission tomography compartmental models: A basis pursuit strategy for kinetic modeling. J. Cerebral Blood Flow \& Metabolism 22, 1425-1439 (2002)

6. Knight, P.A.: Fast rectangular matrix multiplication and qr decomposition. Linear Algebra and its Applications 221, 69-81 (1995)

7. Newton, H.B., Jolesz, F.A. (eds.): Handbook of Neuro-Oncology Neuroimaging. Academic Press, London (2007)

8. Reilhac, A., Batan, G., Michel, C., Grova, C., Tohka, J., Collins, D.L., Costes, N., Evans, A.C.: PET-SORTEO: Validation and development of database of simulated PET volumes. IEEE T. Nuclear Science 52(5), 1321-1328 (2005)

9. Saad, A., Hamarneh, G., Moller, T., Smith, B.: Kinetic modeling based probabilistic segmentation for molecular images. In: Metaxas, D., Axel, L., Fichtinger, G., Székely, G. (eds.) MICCAI 2008, Part I. LNCS, vol. 5241, pp. 244-252. Springer, Heidelberg (2008)

10. Schiepers, C., Chen, W., Cloughesy, T., Dahlbom, M., Huang, S.-C.: ${ }^{18}$ fdopa kinetics and brain tumours. J. Nuclear Medicine 48(10), 1651-1661 (2007)

11. Schiepers, C., Hoh, C.K., Nuyts, J., Wu, H.M., Phelps, M.E., Dahlbom, M.: Factor analysis in prostate cancer: Delineation of organ structures and automatic generation of in- and output functions. IEEE T. Nuclear Science 49(5), 2338-2343 (2002)

12. Sitek, A., Di Bella, E.V.R., Gullberg, G.T., Huesman, R.H.: Correction of ambiguous solutions in factor analysis using a penalized least squares objective. IEEE T. Medical Imaging 21(3), 216-225 (2002)

13. Szczesny, M.: Complexity of initial-value problems for ordinary differential equations of order k. Journal of Complexity 22(4), 514-532 (2006) 